\title{
Having It Both Ways: Consciousness, Unique Not Otherworldly
}

\section{Forthcoming in Philosophia (http://www.springer.com/philosophy/journal/11406)}

\section{Introduction}

Are phenomenal truths a priori deducible from physical truths? A bit more precisely, suppose that ' $\mathrm{P}$ ' stands for the conjunction of all physical facts and laws about the world expressed in the fundamental vocabulary of a true and complete physical theory; 'T' stands for a 'that's all' claim stating that what is expressed in $P$ is the complete description of our world; ' $\mathrm{I}$ ' stands for basic indexical information of the sort, 'I am here' and 'It is now;' and 'Q' stands for any true claim about phenomenal states. Is the conditional $P I T \rightarrow Q$ knowable a prior? ${ }^{1}$

Antiphysicalists say 'no,' and on the basis of this answer, they infer an ontological gap between physical facts and phenomenal facts. Their argument typically runs as follows:

(1) If physicalism is true, then it is necessary that $P I T \rightarrow Q$.

(2) If $P I T \rightarrow Q$ is not knowable a priori, then $P I T \rightarrow Q$ is either false or contingent.

(3) $\quad P I T \rightarrow Q$ is not knowable a priori.

$\therefore$ (4) Physicalism is false.

The Phenomenal Concept Strategy (PCS) is a theoretical attempt to argue that the above argument is unsound. ${ }^{2}$ Premise (2), PCS contends, is false, for the conditional PIT $\rightarrow \mathscr{Q}$ can be both true and necessary and yet not knowable a priori. PCS's argument in support of this conclusion unfolds in two main steps. First, PCS distinguishes between phenomenal and physical or functional concepts. Second, it holds that, although both types of concepts ultimately pick out physical entities, and in some cases they may even co-refer, the two types of

1 For my formulation of this conditional, I rely on Chalmers and Jackson (2001)

2 Proponents of PCS include: Balog (2012); Diaz-Leon (2008) and (2010); Hill (1997); Hill and McLaughlin (1999); Loar (1990), (1997) and (2003); Papineau (2002) and (2006); Perry (2001); and Tye (1995) and (2000). The label 'the phenomenal concept strategy' comes from Stoljar (2005). 
concepts are conceptually independent. That is to say, a priori reasoning alone is insufficient to permit a subject to deduce whether a phenomenal description co-refers with a physical or functional description - even if physicalism requires that the two descriptions do so necessarily.

By admitting conceptual dualism, proponents of PCS resist ontological dualism: a failure of a priori entailment is no longer a symptom of failure of metaphysical necessitation. For proponents of PCS, consciousness is unique, insofar as there is indeed an epistemic gap between PIT and $Q$. Yet its uniqueness is a fact that admits of a physical explanation. The exceptional epistemic status of phenomenal truths is the consequence of the exceptional nature of the concepts involved in such truths, i.e., phenomenal concepts. In other words, proponents of PCS want to have it both ways. Consciousness, they hold, is unique, but not otherworldly.

My objective in this essay is to defend PCS from an objection that purports to undermine it. According to this objection, PCS is faced with a rather troubling dilemma: either the features of phenomenal concepts that PCS posits in order to explain our epistemic situation with regard to consciousness can be explicable in physical terms or not. If not, then PCS, although perhaps useful in elucidating aspects of consciousness, is of no use to theorists who maintain that there is no ontological distinction between consciousness and matter. But if those features of phenomenal concepts can be explicable in physical terms, then they fall short of explaining our epistemic situation with regard to consciousness. If the objection is right, PCS is a theoretical chimera: it is either too good to be physical or too physical to be good.

The objection just rehearsed is found in Chalmers (2006 and 2010) and, in a somewhat different form, in Levine (2006). In what follows, I focus on Chalmers' systematic articulation of the objection. The layout of this essay is as follows: Section 2 presents, explains, and motivates the aforesaid objection to PCS. Sections 3 and 4 provide a novel response to the objection by arguing that the objection itself is faced with a dilemma: either our epistemic situation can be adequately characterized only in phenomenal terms or an adequate characterization of it can also be given in topic-neutral terms. Section 3 shows that if a description of our epistemic situation must be characterized phenomenally, then PCS is not 
undermined by Chalmers' objection. Although the features of phenomenal concepts that PCS posits to account for our epistemic situation with regard to consciousness are not explicable in purely physical terms, PCS does not collapse into a form of dualism. Section 4 argues that if an adequate characterization of our epistemic situation can be given in topic-neutral terms, then, contra Chalmers, PCS can both explain our epistemic situation and be physically explicable. Either way, PCS is safe from Chalmers' objection.

\section{Chalmers' Objection}

Letting ' $\mathrm{C}$ ' stand for the thesis that human beings have certain psychological features, Chalmers (2010, p.311) holds that PCS is committed to the following three claims: (i) It is the case that $C$ obtains; (ii) $C$ is such that it is capable of explaining 'our epistemic situation with regard to consciousness'; and (iii) 'C itself can be explained in physical terms.' Although PCS is committed to all three claims, Chalmers argues that PCS cannot simultaneously meet (ii) and (iii). He writes:

For any candidate thesis $C$ about psychological features of human beings, then either: 1. $C$ is not physically explicable or

2. $C$ does not explain our epistemic situation with regard to consciousness (ibid., p.312).

Assuming that PCS is indeed committed to claims (i) - (iii) and that claims (ii) and (iii) are at odds with each other, then Chalmers' dilemma constitutes a rather serious objection to PCS. Regardless of its specific nature, no account of PCS can both provide support for physicalism and explain our epistemic situation.

My aim in this section is to articulate the arguments that Chalmers advances in support of the two horns of the dilemma. Although in my presentation of Chalmers' position I will also suggest ways in which the proponents of PCS can apply pressure to Chalmers' account, the present section is concerned primarily with matters of exposition. The task of critically 
evaluating Chalmers' position is taken up in the following two sections. In those sections, I provide a systematic response to Chalmers' objection in a way that summarizes and complements many of the extant responses in the literature.

\subsection{Terminological Aside}

Before presenting in detail Chalmers' account, it is necessary to explicate two terms that play a crucial role in it: physical explicability and epistemic situation.

Physical explicability. If $X$ is physically explicable, then there is a reductive explanation of $X$ in physical or microphysical terms. Such an explanation, Chalmers writes, is one 'that makes transparent why some high-level truth obtains, given that certain low-level truths obtain' (ibid., p.313). Elsewhere, Chalmers provides a more detailed description of the nature of this type of explanation by spelling out the idea of transparent explanation in terms of logical supervenience:

A natural phenomenon is reductively explainable in terms of some lower-level properties if the property of instantiating that phenomenon is globally logically supervenient on the low-level properties in question. [...] If the property of exemplifying a phenomenon fails to supervene logically on some lower-level properties, then given any lower-level account of those properties, there will always be a further unanswered question: Why is this lower-level process accompanied by the phenomenon? (Chalmers 1996, p.48; cf. Chalmers and Jackson 2001)

Accordingly, if $C$ is physically explicable then $C$ is shown to be the logical consequence of a set of antecedent physical or microphysical statements. In other words, if $C$ is physically explicable, then $C$ is entailed a priori by PIT. As a result, whatever arbitrariness or mystery surrounds $C$ is replaced by whatever arbitrariness or mystery may surround PIT: '[T] he epistemic contingency in the macroscopic phenomena is reduced to the epistemic contingency in the microphysical phenomena: there is no further epistemic contingency in the connection' (Chalmers and Jackson 2001, p.351). Of course, if by ' $C$ is physically explicable' we simply mean ' $C$ is entailed $a$ priori by PIT', then, according to Chalmers, there is a straightforward way by which we can 
determine whether $C$ is physically explicable. We need to ask whether PIT\& $C$ is conceivable. That is, can we conceive of beings who are physically identical to us but who nonetheless lack whatever features $C$ assumes that we possess? If so, then $C$ is not physically explicable.

Epistemic situation. Chalmers offers two articulations of this term. The first comes very close to identifying epistemic situation with the epistemic gaps that hold between physical truths and phenomenal truths. Chalmers states that in order for $C$ to explain our epistemic situation with regard to consciousness, $C$ must explain 'why we are confronted with the relevant distinctive epistemic gaps' (Chalmers 2010, p.311). ${ }^{3}$

Later on however, Chalmers gives, what appears to be, a different characterization of the term epistemic situation. He writes:

I will take it that the epistemic situation of an individual includes the truth values of their beliefs and the epistemic status of their beliefs (as justified or unjustified, and as cognitively significant or insignificant). As before, an epistemic situation (and a sentence E characterizing it) should be understood in topic-neutral terms, so that it does not build in claims about the presence of phenomenal states or phenomenal concepts. We can say that two individuals share their epistemic situation when they have corresponding beliefs, all of which have corresponding truth values and epistemic status (ibid., p.316).

This second articulation of the term epistemic situation is important for at least two reasons. First, it is intimately connected both to the truth-values and to the epistemic status of beliefs. Accordingly, a subject $S 1$ shares subject S2's epistemic situation if and only if (a) S1 and S2 have corresponding beliefs; (b) the truth-values of their corresponding beliefs are the same; and (c) the corresponding beliefs share their justification and their cognitive significance. Second, in this passage, Chalmers insists that $E$, a sentence characterizing the epistemic situation of an individual, should be given in topic-neutral terms: namely, 'terms that do not explicitly attribute phenomenal states or concepts that refer to them' (ibid., p.314).

\footnotetext{
${ }^{3}$ Presumably, what Chalmers means here is not that an explanation of our epistemic situation with regard to consciousness consists of, and is entirely exhausted by, an explanation of why we face certain epistemic gaps. Rather, I take Chalmers to mean that an explanation of our epistemic situation explains, among other things, why we face those epistemic gaps. I return to this issue in section 4.2.
} 
How does this latter characterization of epistemic situation relate to the former? According to Chalmers, it is crucial to the antiphysicalist arguments that the relevant epistemic gaps are characterized in a way that they invoke the truth-values and epistemic status of beliefs about phenomenal states. He writes:

Recall that the strategy [i.e., PCS] is intended to resist the antiphysicalist's inference from an epistemic gap to an ontological gap by showing how the relevant epistemic gap may exist even if physicalism is true. In the antiphysicalist's arguments, the relevant epistemic gap (from which an ontological gap is inferred) is characterized in such a way that truth and knowledge are essential. For example, it is crucial to the knowledge argument that Mary gains new factual knowledge or at least new true beliefs. It is crucial to the conceivability argument that one can conceive beings that lack phenomenal states that one actually has. And it is crucial to the explanatory gap that one has cognitively significant knowledge of the states that we cannot explain. [...] So truth value is essential to the relevant epistemic gaps (ibid., p.324).

Nonetheless, even if we grant that a description of our epistemic situation with regards to consciousness $(E)$ is one that captures the epistemic gap between $P I T$ and $Q$ and as such, must include the truth-values and epistemic status of our beliefs, this still does not show that $E$ must be given in topic-neutral terms. Is it not the case that our epistemic situation is such that an adequate description of it must refer to phenomenal states? If so, it is hard to see how a topicneutral characterization will be an adequate characterization of our epistemic situation. At this point, I wish to remain noncommittal in regards to whether $E$ should be specified in topicneutral terms or not. I will, however, assume, as Chalmers himself does, that the truth-values and epistemic status of beliefs are 'essential to the relevant epistemic gaps' (ibid.).

\subsection{The First Horn of the Dilemma}

The first horn of the dilemma consists of the claim that $C$ is not physically explicable. Chalmers' argument for this claim is straightforward. Given that ' $C$ is physically explicable' means ' $C$ is entailed a priori by PIT, then $C$ is not physically explicable only if PIT\& $\sim$ is conceivable. That is to say, if 'one can conceive of physical duplicates that lack the key features attributed by thesis 
$C$,' then $C$ is not physically explicable, and an explanatory gap between PIT and $C$ exists (ibid., p.313).

Here, Chalmers' claim is not that one must grant that $P I T \& \sim C$ is conceivable. Rather, he makes a conditional point: insofar as one grants that PIT\& $\sim C$ is conceivable, then one must accept that $C$ is not physically explicable. Of course, in order for this conditional claim to amount to an objection against PCS, one should also add that its acceptance leads to undesirable consequences for PCS. This is precisely what Chalmers does. Assuming that PIT\& C is conceivable, he tells us that:

[T]here will be no wholly physical explanation that makes transparent why $C$ is true. To explain why, in the actual world creatures with the relevant physical structure satisfy thesis $C$, we will need additional explanatory materials, just as we need such principles to explain why actual creatures with this physical structure are conscious (ibid.).

As a result, PCS will have failed to show how the epistemic gaps between phenomenal truths and physical truths are consistent with physicalism: the features of phenomenal concepts that purport to explain our epistemic situation are themselves subject to an epistemic gap.

We can put Chalmers' argument as follows:

(5) If PIT\& $\sim C$ is conceivable, then $C$ cannot be reductively or transparently explained in terms of PIT.

(6) If $C$ cannot be reductively or transparently explained in terms of PIT, then $C$ is not physically explicable.

(7) If $C$ is not physically explicable, then there will be an explanatory gap between physical processes and the relevant features of phenomenal concepts.

(8) If there is an explanatory gap between physical process and the relevant features of phenomenal concepts, then PCS is of no use to physicalists.

$\therefore \quad$ (9) If PIT\& $\sim C$ is conceivable, then PCS is of no use to physicalists. 
Premises (5), (6), and (7) follow straightforwardly from the manner in which I articulated the notion of physical explicability. To be physically explicable, I stated, in line with Chalmers' understanding of this notion, is to be reductively or transparently explained in terms of PIT. That is, if $C$ is reductively explained, then $C$ is entailed a priori by PIT. But if PIT a priori entails $C$, then PIT\& $\sim C$ must not be (ideally, at least) conceivable. Contrariwise, not only is $C$ not physically explicable, but also, there is an epistemic (and explanatory) gap between physical processes and the relevant features of phenomenal concepts posited by $C$. In other words, if PIT\& $\sim C$ is conceivable, then the psychological features posited by PCS in order to explain our epistemic situation are not physically explicable.

The argument can be objected to in at least two ways. First, one may take issue with Chalmers' notion of physical explicability. More specifically, one might deny that a priori entailment is necessary for physical explicability (see author [1]). The most promising way of doing this is to show that there are epistemic gaps even in typical examples of micro-macrophysical explanation, that is, examples where a higher-order property is explained in terms of a lowerlevel set of properties. For instance, one might argue that water facts are not entailed a priori by $\mathrm{H}_{2} \mathrm{O}$ facts, even though water is identical to (or metaphysically necessitated by) $\mathrm{H}_{2} \mathrm{O}$. If micromacrophysical explanations are subject to similar epistemic gaps as psychophysical explanations, then epistemic gaps cannot be taken as indicative of ontological gaps - assuming, of course, that no ontological gap exists between the micro and the macro levels.

A second reaction to the argument is to deny premise (8): even though the antecedent is true, the consequent does not follow (see Papineau 2006; Balog 2012). That is to say, PCS is still of use to physicalists, even if $C$ cannot be reductively explained. In section 3 , I develop a version of this response.

\subsection{The Second Horn of the Dilemma}

The second horn of the dilemma amounts to the conditional claim that if $C$ is physically explicable and thus, PIT\& $\sim C$ is not conceivable, then $C$ does not explain our epistemic 
situation. It is imperative to clarify the circumstances under which PIT\& $\sim$ turns out to be inconceivable. As long as one accepts both that (a) $P I T \& \sim Q$ is conceivable, and (b) $C$ is such that it is true only if one has phenomenal experiences, then one should also grant that PIT\& C is conceivable. Since (a) is a claim that proponents of PCS grant and (b) is a plausible construal of the psychological features posited by $C$, then there is a strong temptation to assert the conceivability of $P I T \& \sim C$ as unavoidable.

Such a reaction, however, would be unduly quick. Although it is part and parcel of PCS that $P I T \& \sim Q$ is conceivable, it is not necessary to construe $C$ in such a way that would require the existence of phenomenal states. A proponent of PCS can instead provide a topic-neutral characterization of $C$. That is to say:

Instead of casting thesis $C$ as a thesis explicitly about phenomenal concepts, one can cast it as a thesis about quasi-phenomenal concepts, where these can be understood as concepts deployed in certain circumstances, that are associated with certain sorts of perceptual and introspective processes, and so on (ibid., p.314).

If phenomenal concepts are understood as quasi-phenomenal concepts, then arguably PIT\& $C$ is not conceivable. Hence, the proponent of PCS who characterizes $C$ in topic-neutral terms can meet the third desideratum of PCS, namely: $C$ is physically explicable, for arguably, under a topic-neutral characterization of $C$, PIT entails a priori $C$. But it is precisely here where Chalmers' second horn of the dilemma becomes pertinent. Physical explicability, according to Chalmers, comes with a price: a topic-neutral characterization of $C$ cannot explain our epistemic situation. Let me explain.

If a topic-neutral characterization of $C$ is granted and $P I T \& \sim C$ is not conceivable, then there is no scenario according to which PIT obtains but $C$ does not. In other words, if PIT\& $\sim$ is not conceivable, then even zombies satisfy $C$. This result provides us with a way to test whether $C$ can explain our epistemic situation: if it turns out that zombies do not share our epistemic situation, then $C$ falls short of explaining our epistemic situation, for $C$ is true for 
zombies. Chalmers' argument for this horn of the dilemma can be stated as follows (see ibid., p.315):

(10) If $P I T \& \sim C$ is not conceivable, then zombies satisfy $C$

(11) Zombies do not share our epistemic situation.

(12) If zombies satisfy $C$ and do not share our epistemic situation, then $C$ cannot explain our epistemic situation

$\therefore \quad$ (13) If PIT\& $\sim C$ is not conceivable, then $C$ cannot explain our epistemic situation.

The crucial premises of the argument are (11) and (12). Regarding (12), Chalmers says very little. In fact, he merely states that it 'is simply another application of the connection between conceivability and explanation' (ibid., p.316). Presumably, what Chalmers means by this is the following: If $C$ is such that zombies satisfy it, then the features posited by $C$ must not include, depend on, or utilize phenomenal states in any way. But assuming (11), there must be something more to our epistemic situation that is not explained by a topic-neutral characterization of $C$. Thus, a topic-neutral characterization of $C$ cannot explain our epistemic situation.

Yet, it is not entirely clear why a topic-neutral characterization of $C$ would not be capable of explaining our epistemic situation, even if we do not share our epistemic situation with zombies. Let ' $\mathrm{E}$ ' stand for a sentence describing our epistemic situation, and let ' $\mathrm{E}_{\mathrm{Z}}$ ' stand for a sentence describing the epistemic situation of zombies. The conjunction of (i) the propositions expressed by $E$ and $E_{Z}$ are not one and the same, and (ii) $C$ explains (i.e., a priori entails) $E_{Z}$, does not entail that $C$ does not explain $E$. Why couldn't $C$ explain both $E$ and $E_{Z}$ and the further fact that $E$ and $E_{Z}$ are not the same? Chalmers would be correct to infer (12) if either an adequate characterization of our epistemic situation has to be given only in phenomenal terms, or, what amounts to the same thing, the difference between $E$ and $E_{Z}$ could not be captured in topic-neutral terms. Since a topic-neutral characterization of $C$ explains $E_{Z \text {, }}$ 
then to assume either option is to reject the contention that $E$ can be adequately captured in topic-neutral terms. But recall that Chalmers earlier insisted that 'epistemic situation (and a sentence $E$ characterizing it) should be understood in topic-neutral terms, so that it does not build in claims about the presence of phenomenal states or phenomenal concepts' (ibid., p.324). If $E$ is characterized topic-neutrally, then I do not see why $C$ (which is also given in topicneutral terms) could not explain E. I return to this issue in subsection 4.2.

In support of (11), Chalmers provides two distinct sets of considerations. First, Chalmers considers certain statements uttered by zombies and concludes that the most natural reading of those utterances betrays the fact that zombies do not share our epistemic situation. For instance, Chalmers asks how should we understand the sentence, 'I am phenomenally conscious,' when uttered by a zombie. Although the content of the utterance might be unclear to us, Chalmers suggests that it is plausible that zombies assert something false. Had we, however, uttered the same sentence we would be expressing something true. Given that (at first sight, at least) the same utterance yields a different truth-value when uttered by us than when uttered by zombies, zombies cannot share our epistemic situation. As Chalmers himself admits however, this argument is fairly contentious. How can we be sure that what zombies express is false, if we cannot be sure about the content of their utterance? It is for this reason that Chalmers finds recourse to a second argument in support of (11).

Chalmers' second argument compares the epistemic progress that Mary does when released from her black-and-white room to that of Zombie Mary's when she too exits her black-and-white room. (Zombie Mary is physically identical to Jackson's Mary, but not phenomenally conscious.) Chalmers argues that 'there is no reason to believe that Zombie Mary will gain cognitively significant introspective knowledge, analogous to the cognitively significant knowledge that Mary gains' when they both come to perceive a red object (Ibid., p.318). He adds: 
There is nothing for Zombie Mary to gain knowledge of. For Zombie Mary, all is dark inside, so even confronting her with a new sort of stimulus will not bring about new significant introspective knowledge (ibid.).

Therefore, Mary and Zombie Mary do not share the same epistemic situation. But since Mary shares our epistemic situation, then zombies do not share our epistemic situation.

One can object to Chalmers' argument in support of the latter horn of the dilemma by denying either (11) or (12). By denying (11), one asserts that zombies do in fact share our epistemic situation (see Papineau 2006; Carruthers and Veillett 2007; Levin 2008, p.409). Insofar as that is the case, and since zombies satisfy $C$ and $C$ is physically explicable, then PCS can both explain our epistemic situation and support physicalism. Alternatively, one may wish to deny the conditional claim made by (12). That is, one may argue that a topic-neutral characterization of $C$ suffices to explain our epistemic situation, even if zombies do not share our epistemic situation.

\section{The Response: First Part}

In the previous section, I hinted at the various ways in which proponents of PCS can resist Chalmers' argument. My aim in the following two sections is to develop these responses in a systematic manner. I will do by arguing that Chalmers' objection is itself faced with a dilemma. Either the description of our epistemic situation, $E$, should be given in topic-neutral terms or it should be specified in a way that it refers to phenomenal states and implicates phenomenal concepts. Either way, I shall argue, PCS is not undermined.

In the present section, I show how PCS can respond to Chalmers' objection, if we accept that $E$ should be given a phenomenal characterization. In the following section, I argue that PCS can provide a response to Chalmers' objection, even if $E$ is given a topic-neutral characterization.

\subsection{A Phenomenal Characterization of our Epistemic Situation}


Under the assumption that $E$ should be given a phenomenal characterization, then either $C$ reductively explains $E$ or it does not. Suppose the latter. If $C$ does not reductively explain $E$, then $C$ is not given in phenomenal terms. Perhaps, it is given in topic-neutral terms. But if $C$ is given a topic-neutral characterization, then arguably PIT reductively explains $C$. Is there a problem with this result? According to Chalmers there is. Although it is physically explicable, $C$ no longer explains our epistemic situation.

On closer examination, however, it is clear that Chalmers cannot demand that $C$ must explain our epistemic situation. $C$ explains our epistemic situation $E$, only if $C$ a priori entails $E$. But under the supposition that $E$ is given in phenomenal terms, $C$ can a priori entail $E$, only if $C$ a priori entails truths about consciousness. That is, $C$ (which is given in topic-neutral terms) explains our epistemic situation (which is given in phenomenal terms), only if phenomenal truths are a priori entailed by PIT. Since it is a premise of PCS, that PIT does not a priori entail Q, Chalmers cannot demand that $C$ reductively explains $E$, when $E$ is expressed in phenomenal terms. To do so, it is to simply reject PCS from the start.

A similar story can be told in case that we assume that $C$ reductively explains $E$, when $E$ is given in phenomenal terms. If $C$ reductively explains $E$, then $C$ a priori entails $E$. If that is so, then $C$ cannot be given a topic-neutral characterization. For $C$ to obtain - that is, for it to be the case that we actually have the psychological features posited by $C$ - we must have phenomenal consciousness. What this supposition amounts to is that a reductive explanation of $C$ is possible only if there is a reductive explanation of consciousness. But just like before, to demand that PIT reductively explains $C$ (when $C$ is given a phenomenal characterization) is to demand that PIT a priori entails $Q$.

Regardless of whether $C$ reductively explains $E$, the conclusion is this: assuming that $E$ should be given a phenomenal characterization, Chalmers' argument does not add anything new to the physicalist-antiphysicalist dialectic. It merely pushes the explanatory gap that PCS already admits that exists between consciousness and physical processes to a different level. By assuming that $E$ must be given a phenomenal characterization, the explanatory gap between 
PIT and $Q$ is now also found between $C$ and $E$ (if $C$ is characterized topic-neutrally) or between PIT and $C$ (if $C$ is characterized phenomenally). What gives rise to these explanatory gaps is that which also gives rise to the original explanatory gap between PIT and Q: namely, the fact that phenomenal experiences, by being conceptualized by phenomenal concepts, preclude a reductive explanation. And what explains how those explanatory gaps are compatible with physicalism is that which also explains how an explanatory gap between PIT and $Q$ is compatible with physicalism: namely, the fact that phenomenal and physical/or functional concepts can pick out the same physical states, even if the two types of concepts are conceptually independent.

The provided response bears similarities to both Papineau's (2006) and Balog's (2012) respective responses to Chalmers' objection. This, I think, is only natural, for as I have shown in the previous section, there are only a certain number of objections that one can launch against Chalmers' position. Having said that, my response differs from Papineau and Balog's in at least three crucial respects. First, my response is conditional on the acceptance of the claim that our epistemic situation should be characterized phenomenally. I do not insist that our epistemic situation must be characterized phenomenally. Instead, I argue that in case that it is characterized so, PCS can respond to Chalmers' objection. Second, unlike Papineau and Balog's responses, which rest on the acceptance of a rather specific (and perhaps, contentious) account of the nature of phenomenal concepts, my response remains non-committal with regard to the nature of phenomenal concepts and how phenomenal concepts refer. For that reason, if successful, my response constitutes an important contribution to the dialectic between proponents of PCS and Chalmers since it illustrates that one can respond to Chalmers' objection even if one does not commit himself/or herself to a particular account of phenomenal concepts. Finally, in the remainder of this section, I consider a number of objections to the provided response and argue that there are no good reasons to think that it 
cannot succeed. Since some of the objections that I consider have not been addressed by either Papineau (2006) or Balog (2012), my presentation goes beyond those responses. It also contributes to and strengthens their respective positions. ${ }^{4}$

\subsection{Objections and Replies}

\subsubsection{Justifying Physicalism's Compatibility With an Explanatory Gap}

One might object that proponents of PCS need to do something more than simply assert that the explanatory gap between PIT and $C$ (if $C$ is characterized phenomenally) or that between $C$ and $E$ (if $C$ is characterized topic-neutrally) is compatible with physicalism. Chalmers explains:

[T] he proponent [of PCS] may respond by saying that ontological physicalism is compatible with the existence of explanatory gaps. But now we are back where we started, before the phenomenal concept strategy came in. Antiphysicalists argue from an epistemic gap to an ontological gap. The phenomenal concept strategy [...] was supposed to ground the rejection of this inference, by showing how such epistemic gaps can arise in a purely physical system. If successful, the strategy would help to justify the claim that the epistemic gap is compatible with ontological physicalism, and so would lend significant support to type B-materialism. But the weaker version of the strategy [one which simply asserts that explanatory gaps are compatible with physicalism] [...] can give no such support. On this version, the proponent needs independent grounds to reject the inference from an explanatory gap to an ontological gap (ibid., pp.321-2).

Chalmers' contention is that PCS needs to 'justify the claim that the epistemic gap is compatible with ontological physicalism' (ibid., p.322). Only then can PCS be assumed to provide support for the type of physicalism that admits an explanatory gap between physical processes and consciousness.

\footnotetext{
4 As far as I know, no other paper addresses Chalmers' most recent formulation of his objection to the phenomenal concept strategy (see Chalmers 2010). In The Character of Consciousness, Chalmers revisits his 2006 objection to the phenomenal concept strategy and responds to Papineau (2006), Balog (2012), and Diaz-Leon (2010). In what follows, I consider Chalmers' responses to the aforesaid papers and show that they are unsuccessful.
} 
Reply: PCS does provide justification for the claim that an epistemic gap between physical truths and phenomenal truths is not symptomatic of an ontological gap. It does so by showing that there is a physicalistically acceptable explanation of why an epistemic gap between physical truths and phenomenal truths does not lead to an ontological gap between physical facts and phenomenal facts. According to PCS, psychophysical identities (or instances of psychophysical metaphysical necessitation) cannot be known a priori because (a) such psychophysical identities (or instances of metaphysical necessitation) involve both phenomenal and physical or functional concepts, and (b) phenomenal concepts are conceptually isolated from physical or functional concepts. As long as there is a physicalistically acceptable account of what gives rise to conceptual independence, then PCS does provide support for physicalism, even if epistemic or explanatory gaps are admitted.

I anticipate two counter-objections to this reply.

Counter-objection 1: It is not sufficient for PCS to hold that there is a physicalistically acceptable account of conceptual independence. Instead, what PCS needs to demonstrate is the claim that there is a reductive explanation of conceptual independence. Otherwise, a new explanatory gap will arise - this time between PIT and the features that purport to account for conceptual independence. But if there is an explanatory gap between PIT and the features that account for conceptual independence, then conceptual independence cannot provide justification for the claim that the explanatory gap between PIT and $Q$ (or that between PIT and $C$, or that between $C$ and $E$ ) is compatible with physicalism. In that case, a different explanation must be provided: one that also justifies the claim that the explanatory gap between PIT and the features that account for conceptual independence is compatible with physicalism.

Reply: Proponents of PCS should not oppose the claim that the features that account for conceptual independence need to be reductively explained. In fact, some proponents of PCS have already advanced explanations of conceptual independence that admit to a reductive explanation. For instance, Loar (2003, p.116), in response to the question, how should we explain the a posteriori status of psychophysical identities?, argues that the explanation consists in the fact that 
the concepts involved in psychophysical identities 'are not cognitively tied in a certain way.'

Loar goes on to spell out the notion of being cognitively tied in a psychological manner:

The ordinary pain concept we may suppose to have a special connection with neural pain centers; and the theoretical concept of c-fiber stimulation a special connection with verbal theoretical centers. It would hardly be surprising if neural relations do not support a direct cognitive connection (ibid., p.117).

In a more recent paper, Loar elaborates on how the a posteriori status of psychological identities can be accounted by phenomenal concepts' inferential isolation:

... being a posteriori is not a modal-semantic property but a psychological-cognitive property, or to put it another way, a matter of conceptual role. The conceptual role of the phenomenal concept feeling like that, and the conceptual role of the verbal-theoretical concept of c-fiber stimulation are conceptually independent. That is all one needs to explain the a posteriori status of the identity. The phenomenal concept of feeling pain and the theoretical concept of c-fiber stimulation are such empirically independent sorts of concept that the metaphysics of their reference are, in the face of it, beside the point (Loar 2007, p.452).

According to Loar's account, the conceptual isolation of phenomenal concepts is the product of how certain psychological or cognitive mechanisms are manifested. ${ }^{5}$ But if conceptual independence can be psychologically or cognitively explained, then conceptual independence can also be reductively explained. In other words, even zombies' quasi-phenomenal concepts will be conceptually independent of their physical or functional concepts. Similarly to our phenomenal concept pain, the quasi-phenomenal concept pain* of a zombie will too have 'a special connection with neural pain centers' (Loar 2003, p.117). Given that the zombie's

\footnotetext{
${ }^{5}$ Suppose that the neural realizers of phenomenal concepts are directly connected to the neural realizers of phenomenal experiences. In turn, suppose that the neural realizers of physical or functional concepts are directly connected to the neural realizers of theoretical thinking, reasoning, or verbal processing. If it turns out that the neural realizers of phenomenal concepts are not connected in any direct fashion to the neural realizers of physical or functional concepts then this would explain the conceptual isolation of the two types of concepts. When we subsume a phenomenal experience under a phenomenal concept, we deploy a certain kind of concept. In so doing, we activate a certain neurological structure - one which is not connected in any direct way to the neurological structure of the mechanism(s) responsible for the deployment of physical or functional concepts. (Similar claims can be made about the reference-fixing mechanisms of the two types of concepts: they are isolated insofar as there are no direct connections between the neural realizers of those mechanisms.)
} 
theoretical concept c-fiber stimulation will also have a 'special connection with verbal theoretical centers' and that the neural relations between the two centers 'do not support a direct cognitive connection,' then the zombie's quasi-phenomenal concept pain* will be conceptually isolated from his/her theoretical concept c-fiber stimulation, even if the two types of concepts are coreferential (ibid.). (For a similar account of conceptual independence, see Hill and McLaughlin 1999.) What this shows is that conceptual independence is reductively or physically explicable: it is inconceivable that PIT obtains but conceptual independence does not.

It is worth noting Chalmers' own position on this matter. He admits that conceptual independence is a feature of phenomenal concepts that can be reductively explainable. He writes: 'it is plausible that a zombie's physical and quasi-phenomenal beliefs are no more inferentially connected than a conscious being's beliefs' (Chalmers 2010, p.325). The fact that Chalmers admits that inferential isolation can be a feature both of phenomenal beliefs and of quasi-phenomenal beliefs makes it clear that conceptual or inferential isolation does not depend on phenomenal experiences. Therefore, the claim that what explains conceptual independence between phenomenal concepts and physical or functional concepts needs to be reductively explained is no threat to PCS. PCS can and should provide a purely psychological or cognitive explanation of phenomenal concepts' (and as a consequence, of phenomenal beliefs') conceptual independence. Since this conceptual independence is that which accounts for the epistemic and explanatory gap between PIT and $Q$, PCS has provided justification for how such epistemic and explanatory gaps are compatible with physicalism.

Counter-objection 2: PCS need not only provide justification for the claim that the explanatory gap between PIT and $Q$ is compatible with physicalism, it also needs to provide justification for the further claim that the explanatory gaps between $P I T$ and $C$ (if $C$ is characterized phenomenally) or between $C$ and $E$ (if $C$ is characterized topic-neutrally) are compatible with physicalism. For even if conceptual independence can be reductively explained, the gaps between PIT and $C$ or between $C$ and $E$ still remain. Without a justification of why the epistemic gaps between PIT 
and $C$ or between $C$ and $E$ are in line with physicalism, Chalmers will insist that PCS has failed to provide support for physicalism.

Reply: Can PCS provide justification for the claim that the explanatory gaps between PIT and $C$ or between $C$ and $E$ are compatible with physicalism? It can. In fact, if I am right to insist that those explanatory gaps are caused by the same features of phenomenal concepts that cause the explanatory gap between PIT and $Q$, then what justifies the compatibility of physicalism with a gap between $P I T$ and $Q$ is also what justifies its compatibility with the existence of epistemic gaps either between PIT and $C$ or between $C$ and E. What explains the gap between PIT and $C$ (when $C$ is phenomenally conceived) or the gap between $C$ and $E$ (when $C$ is characterized in topic-neutral terms) is precisely what explains the gap between PIT and $Q$ : namely, the inferential isolation of phenomenal concepts. Given that conceptual independence can be reductively explained, PCS can offer justification for the claim that physicalism is compatible with the explanatory gaps between PIT and $C$ or between $C$ and E. Although PCS bridges neither gaps, it does provide a physical explanation of the existence of those gaps. It shows how those explanatory gaps are the consequences of certain psychological mechanisms. ${ }^{6}$

\subsubsection{Circularity Objection I}

Chalmers summarizes the type of response that I have provided as follows:

\footnotetext{
6 Perhaps, Chalmers would argue that it is not enough for PCS to show that conceptual independence between physical truths and phenomenal truths is compatible with physicalism. What PCS needs to show in addition is that the truth-and-knowledge involving epistemic gaps (and not just conceptual independence) is compatible with physicalism. My response to this objection amounts to a re-iteration of the dilemma that Chalmers' position faces. Conceptual independence between physical and phenomenal truths is not enough to respond to antiphysicalist arguments only if (i) a response to those arguments requires proponents of physicalism to show that PIT a priori entails those truth-and-knowledge involving epistemic gaps and (ii) those truth-and-knowledge involving epistemic gaps are given in phenomenal terms. But as I have argued, an acceptance of (ii) amounts to an acceptance of the claim that $E$ - the description of our epistemic position should be given in phenomenal terms. Once we accept that our epistemic situation must be given a phenomenal description, then it is illegitimate to demand from PCS to explain those gaps. An explanation of those gaps would be possible only under the assumption that consciousness is reductively explained. But in this context, Chalmers cannot demand that consciousness must be reductively explained. Such a demand is tantamount to the rejection of PCS. Chalmers' objection is supposed to show why PCS does not work, not assume its falsity from the very beginning. I am grateful to $\mathrm{X}$ for pressing me to address this worry.
} 
[A]n opponent might suggest that the second-order explanatory gap [the gap between PIT and $C$ or between $C$ and $E$ ] can be explained in terms of the same second-order features of phenomenal concepts that explain the first-order explanatory gap [the gap between PIT and Q] (ibid., p.321).

He quickly dismisses it. The provided response, he writes, 'obviously...leads to a circular explanation' (ibid.). He adds:

Explanatory structures of this sort can be informative but again they will do nothing to deflate the explanatory gap unless the chain of explanation is at same point grounded in physical explanation (ibid.).

Reply: Chalmers' reaction is not entirely transparent to me. For one, he simply states that the provided response is circular, without articulating why the purported circularity is problematic for PCS. Chalmers' assertion that the response is circular does not, at least by itself, constitute an objection. Instead, what he needs to show in order to object to the provided response, is that the response is viciously circular. But there is nothing viciously circular about the explanation provided: features of phenomenal concepts that explain the first gap can explain the second gap as well. (Papineau 2006 and Balog 2012 also make a similar point.) In fact, given that the cause of the explanatory gap between PIT and $C$ (or between $C$ and $E$ ) is the same as the cause of the explanatory gap between PIT and $\mathcal{Q}$, one would expect that whatever accounts for the first gap would also account for the same gap. That is precisely what PCS shows.

Furthermore, a proponent of PCS can agree with Chalmers' claim that at some point the chain of explanation should be grounded in a physical explanation. Indeed, PCS does hold that what explains the existence of an explanatory gap - namely, the inferential isolation of phenomenal concepts - admits of a reductive explanation. What PCS does not offer, of course, is a reductive explanation of phenomenal consciousness. It is an assumption of PCS that phenomenal consciousness cannot be reductively explained. Thus, Chalmers cannot require PCS to offer a reductive explanation of phenomenal consciousness. That is to say, the demand 
that 'the chain of explanation' must be 'at some point grounded in physical explanation' does not apply to phenomenal consciousness itself (ibid.).

\subsubsection{Circularity Objection II}

In the latest version of his objection against PCS, Chalmers advances an additional reason why the type of response offered in this section is problematic. Recall that, in this section, I have argued that in case our epistemic situation is described in phenomenal terms, then proponents of PCS can hold that what explains the explanatory gap between PIT and $C$ or that between $C$ and $E$ is the same as that which explains the gap between PIT and $Q$. Chalmers objects. He writes:

But the phenomenological explanation [i.e., the response to Chalmers' argument offered in this section] is a 'physicalistic explanation' only if we assume as part of the explanation the key claim that phenomenal states are physical states. If we do, then the explanation cannot add support to the key claim. If we do not, then likewise the explanation cannot add support to the key claim (2010, p. 322, n.3).

What Chalmers is getting at in this passage is the following. In order for the explanatory gap between PIT and $Q$ to explain the explanatory gap between PIT and $C$ (or that between $\mathrm{C}$ and E), phenomenal states must be assumed to be physical states. But if we assume that they are physical, then we no longer need PCS. If we do not assume that they are physical, then PCS is useless, for it cannot provide support for the claim that phenomenal states are physical. ${ }^{7}$

Reply: In the quoted passage, Chalmers misconstrues the theoretical obligations of PCS. PCS is meant only as a response to epistemic arguments against physicalism. Specifically, its objective is to show how physicalism is compatible with the existence of certain epistemic gaps. PCS does not purport to provide positive support for physicalism. Other arguments are meant

\footnotetext{
7 I would like to thank $\mathrm{X}$ for presenting this objection to me.
} 
to do so. ${ }^{8}$ Hence, PCS can and does assume that phenomenal states are physical states. Of course, by doing so, it does not automatically respond to epistemic arguments against physicalism. What it has to show in addition is that the existence of epistemic and explanatory gaps between phenomenal truths and physical truths is compatible with the assumption that phenomenal states are physical states. By demonstrating that the two are compatible, PCS concludes that the best conclusion to draw from the observation that there are epistemic gaps is not property (or ontological) dualism but rather conceptual dualism. (The fact that there are arguments for physicalism helps to establish this conclusion).

Hence, contrary to what Chalmers states, PCS can assume that phenomenal states are physical. By assuming that to be the case, PCS is not however rendered superfluous. PCS still needs to demonstrate that those gaps are consistent with physicalism.

\subsection{Summary}

In this section, $I$ argued that under the assumption that $E$ must be given a phenomenal characterization, Chalmers' objection fails to spell trouble for proponents of PCS. If $E$ is characterized in a phenomenal manner, then proponents of PCS have a choice. Either $C$ explains our epistemic situation, and PIT does not explain $C$. Or, PIT does explain $C$, but $C$ fails to explain E. Either way, an additional explanatory gap will emerge. Nonetheless, this additional gap is not only compatible with physicalism, but PCS can also provide justification why that is so. To repeat, what gives rise to the explanatory gap between PIT and $C$ (if $C$ explains $E$ ) or between $C$ and $E$ (if $C$ does not explain $E$ ) is also that which gives rise to the explanatory gap between PIT and Q, namely, the conceptual independence of phenomenal concepts of physical/or functional concepts. Since PCS can provide a reductive explanation of the

8 The most important argument in favor of physicalism is the so-called "causal argument," which holds (roughly) that all physical effects have physical causes and thus everything that has physical effects (including experiences) must be physical. For more on this argument, see Kim (1998); Papineau (2001) and (2002); Yablo (1992); Sturgeon (2000); Stoljar (2010); Tye (2009, chapter 2). 
conceptual independence of phenomenal concepts, PCS has a response to Chalmers' objection. In showing that the cause of the explanatory gaps admits to a reductive explanation, PCS shows that we have good reasons to resist the antiphysicalists' contention that the best explanation of the existence of those gaps is the existence of an ontological gap. Physicalism can be true, even if explanatory gaps persist. And although PCS does not bridge the gap between PIT and $C$ or that between $C$ and $E$, it does provide a physical explanation of why these gaps are unbridgeable.

\section{The Response: Second Part}

\subsection{A Topic-Neutral Characterization of Our Epistemic Situation}

So far, I argued that under the assumption that $E$ should be given a phenomenal characterization, PCS has the resources to respond to Chalmers' objection. Of course, Chalmers can deny that $E$ should be characterized phenomenally. Instead, he can maintain that a description of our epistemic situation should be given in topic-neutral terms. In this section, I argue that even if $E$ is characterized topic-neutrally, proponents of PCS can still respond to Chalmers' objection.

Assuming that our epistemic situation is characterized topic-neutrally, then proponents of PCS have an available response to Chalmers' objection. They can reject Chalmers' contention that zombies do not share our epistemic situation. If $E$ is given in topic-neutral terms, then the truth-values and epistemic status of the respective beliefs of Mary and Zombie Mary should not depend on the presence of phenomenal consciousness, nor should they require phenomenal concepts. If that is so, proponents of PCS can argue that there is no reason to think that the epistemic situation of the two differs. If Mary and Zombie Mary share their epistemic situation, and $C$ explains Zombie Mary's epistemic situation, then it also explains Mary's situation. As a consequence, it explains our epistemic situation as well. 


\subsection{A Difference in Epistemic Situation?}

Chalmers denies that the epistemic situation of Zombie Mary is the same as that of Mary. In extension, he denies that we share the epistemic situation of zombies. He explains:

Let us focus on the epistemic situation of Mary upon seeing red for the first time. Here, Mary gains cognitively significant knowledge of what it is like to see red, knowledge that could not be inferred from physical knowledge. What about Mary's zombie twin, Zombie Mary? What sort of knowledge does Zombie Mary gain when she emerges from the black-and-white room? [...] There is no reason to believe that Zombie Mary will gain cognitively significant introspective knowledge analogous to the cognitively significant knowledge that Mary gains. On the face of it, there is nothing for Zombie Mary to gain knowledge of. For Zombie Mary, all is dark inside, so even confronting her with a new sort of stimulus will not bring about new significant introspective knowledge (ibid., p.318).

Chalmers makes two important claims in this passage. First, Mary's epistemic situation differs from that of Zombie Mary's, insofar as only the former 'gains cognitively significant knowledge of what it is like to see red' when she exits the room (ibid.). Second, the reason why Zombie Mary does not gain cognitively significant knowledge, Chalmers tells us, is that 'for Zombie Mary, all is dark inside' (ibid.). From what Chalmers states in this passage it is tempting to understand the notion of cognitive significance as a characterization of how the subject has come to have certain beliefs and know certain facts. Indeed, in the quoted passage Chalmers characterizes this type of knowledge as introspective. Consequently, we can say that a subject acquires cognitively significant knowledge of what it is like to experience red, only if the subject has come to have an experience of red and the subject has phenomenal awareness of his or her experience. On the contrary, cognitively insignificant knowledge of a phenomenal state is knowledge which has neither being brought about nor is accompanied by the relevant introspective experience.

If we take Chalmers on his word and accept that the reason why Zombie Mary does not have cognitively significant knowledge is because she lacks phenomenal consciousness - i.e., because 'it is all dark inside' - then her epistemic situation will differ from that of Mary, only 
under the assumption that an adequate characterization of Mary's epistemic situation (and in extension, ours) must implicate phenomenal states and phenomenal concepts (ibid.). But in this part of the paper we are assuming that Mary's epistemic situation should be given a topicneutral characterization. Consequently, it is unclear why one should accept Chalmers' conclusion that the epistemic situations of Zombie Mary and Mary differ. Shouldn't a topicneutral characterization of Mary's epistemic situation eschew phenomenal language? If so, it seems that whatever topic-neutral characterization of Mary's epistemic situation we provide, Zombie Mary will satisfy it too.

Chalmers anticipates such a response. He writes:

The $[\ldots]$ reaction is to assert that zombies share our epistemic situation. Where we have beliefs about consciousness, zombies have corresponding beliefs with the same truth values and the same epistemic status. And where Mary acquires new phenomenal knowledge on seeing red for the first time, Zombie Mary acquires new knowledge of a precisely analogous sort. If this is right, then the crucial features of phenomenal concepts might simultaneously be physically explicable and able to explain our epistemic situation (ibid., p.326).

This proposal might be developed in two different ways: either by deflating the phenomenal knowledge of conscious beings or by inflating the corresponding knowledge of zombies. That is, a proponent may argue either that Mary gains less new knowledge than I suggested earlier or that Zombie Mary gains more new knowledge than I suggested earlier. Earlier I argued that Mary gains new, cognitively significant nonindexical knowledge, whereas Zombie Mary does not. The deflationary strategy proposes that Mary gains no such knowledge; the inflationary strategy proposes that Zombie Mary gains such knowledge, too (ibid., p.327).

Ultimately, Chalmers rejects both ways of developing the response. If we deflate Mary's epistemic progress, then we are committed to holding 'that the only new factual knowledge that Mary gains upon seeing red for the first time is indexical knowledge' (ibid.). Chalmers rejects that conclusion, and I agree with him: there is more to Mary's epistemic progress than the mere acquisition of indexical knowledge. If, on the contrary, we inflate Zombie Mary's epistemic progress, then we are committed to holding that: 
[Just as Mary gains cognitively significant nonindexical knowledge involving phenomenal concepts, Zombie Mary gains analogous, cognitively significant nonindexical knowledge involving schmenomenal concepts. So where Mary gains significant knowledge of the form tomatoes cause such-and-such phenomenal state, I am in suchand-such phenomenal state, and this state is such-and-such phenomenal state, Zombie Mary gains significant knowledge of the form tomatoes cause such-and-such schmenomenal state, I am in such-and-such schmenomenal state, and this state is such-and-such schmenomenal state (ibid., pp.327-8).

(To clarify, schmenomenal concepts are the concepts which zombies employ to conceptualize their schmenomenal states, where 'schmenomenal states are not phenomenal states, but they play a role in zombies' lives that is analogous to the role that phenomenal states play in ours' [ibid., p.326]).

Chalmers provides two related reasons in support of his rejection of the inflationary strategy. First, the inflationary response, Chalmers tells us, mischaracterizes zombie scenarios. Although zombies can have some sort of introspective knowledge, that knowledge is not analogous to our introspective phenomenal knowledge. As he writes:

$[\mathrm{W}]$ hen we conceive of zombies, we are not conceiving of beings whose inner life is as rich as ours but different in character. We are conceiving of beings whose inner life is dramatically poorer than our own. And this difference in inner lives makes for dramatic difference in the richness of our introspective knowledge. Where we have substantial knowledge of our phenomenal inner lives, zombies have no analogous introspective knowledge: there is nothing analogous for them to have introspective knowledge of (ibid., p.328).

But Chalmers adduces an additional argument against the inflationary strategy. He argues that even if it is conceivable that zombies have such analogous introspective knowledge, it is also conceivable that they lack it. 'And this latter conceivability claim,' he adds, 'is all that the argument against the phenomenal concept strategy needs' (ibid., p.329). The fact that it is conceivable that zombies lack the assumed analogous introspective knowledge, even when PIT\& $\sim \mathrm{C}$ is inconceivable, shows that $C$ does not reductively explain the presence of such introspective knowledge. Assuming that reductively explaining (the presence of) such 
introspective knowledge is required in order to reductively explain our epistemic situation, then $C$ cannot reductively explain our epistemic situation. Or so Chalmers maintains. ${ }^{9}$

Although Chalmers' response might be applicable to certain defenses of PCS (Carruthers and Veillet 2007; Papineau 2006), it is inapplicable when it comes to my position. This is a virtue of my response to Chalmers' objection. By formulating the response in terms of a dilemma, I manage to avoid problems that other responses face. Let me explain. In my response, I make no claims regarding the type or amount of knowledge that Zombie Mary gains when she exits her black-and-white room. In that respect, I am not inflating in any way Zombie Mary's knowledge. My argument is concerned primarily with Mary's (and consequently, our) epistemic situation, and with the terms that are appropriate to describe it. In the previous section, I considered the ramifications of assuming that $E$ should be given a phenomenal characterization. In this section, I am considering what happens if $E$ is assumed to be given a topic-neutral characterization (see also Levin 2008, pp. 406-10).

Lest I be misunderstood, I should emphasize that a characterization of Mary's epistemic situation in topic-neutral terms does not preclude that Mary acquires introspective (i.e., cognitively significant) phenomenal knowledge when she exits her room. Mary can acquire such knowledge, even if such knowledge cannot be captured by a topic-neutral description. Instead, it is the claim that Mary's epistemic situation can be adequately captured by a topic-neutral description that precludes the acquisition of such knowledge. And in this section, it is precisely this latter and stronger claim that I am assuming. That is to say, the question that Chalmers' objection faces is not whether a topic-neutral characterization of our (and Mary's) epistemic

9 I am not convinced by Chalmers' argument. The conceivability claim under question - i.e., the claim that it is conceivable that zombies lack introspective knowledge even if they have $C$ - can lead to the conclusion that zombies do not share our epistemic situation only if the following two claims are granted: (a) we have introspective knowledge of the sort that zombies lack; and (b) such introspective knowledge cannot be captured in topic-neutral terms. Given, however, that in this section of the paper we are assuming that our epistemic situation, $E$, can be adequately characterized in topic-neutral terms, then we should expect that such introspective knowledge can be captured in topic-neutral terms. In other words, the assumption that $E$ can be adequately characterized in topic-neutral terms furnishes us with reasons to deny (b). Consequently, we should deny Chalmers' claim that zombies do not share our epistemic situation. For more on this issue, see note 10 . 
situation is possible. Rather, the question is whether a topic-neutral characterization is appropriate or adequate. If an adequate characterization of Mary's epistemic situation can be given in topicneutral terms, then we have little reason to think that Mary has acquired any sort of knowledge that her zombie twin cannot also acquire. Of course, one can deny that Mary's epistemic situation can be adequately captured by neutral-topic terms. To do so, however, it is to embrace the first horn of the dilemma that Chalmers' objection faces: namely, that $E$ must be given a phenomenal characterization. As I have argued in the previous section, Chalmers' argument does nothing to undermine PCS in case $E$ is characterized phenomenally.

Hence, if we assume that Mary's epistemic situation can be given an adequate characterization in topic-neutral terms, then there is really no need to inflate Zombie Mary's knowledge in any way. So Chalmers' criticisms against the inflationary strategy are irrelevant. But do we have to deflate Mary's knowledge? We do, but that follows by assumption. In other words, in responding to Chalmers' objection I do not argue that it is correct to deflate Mary's knowledge. Instead, I am merely making a conditional claim: if $E$ should be characterized topicneutrally, then Mary's knowledge, is, ex hypothesi, deflated. Insofar as this is the case, my argument is also not susceptible to Chalmers' criticism of the deflationary strategy. In fact, I think Chalmers is right to insist that what Mary acquires when she exits her room is cognitively significant knowledge. Such a contention, however, does not affect the conditional claim for which I argue. At most, it shows that we should not assume that Mary's epistemic situation can be adequately captured by a topic-neutral description. But if one denies that Mary's epistemic situation can be given an adequate topic-neutral characterization, then one is committed to accepting that $E$ must be described phenomenally.

Finally, suppose that one insists that what 'E' should stand for is not a sentence that describes our entire epistemic situation with regards to consciousness, but rather for a sentence that captures the distinctive epistemic gaps that we face. Just like before, we have a choice as to how to understand these epistemic gaps. Either those epistemic gaps can be adequately characterized topic-neutrally or not. If the former, then Mary and Zombie Mary will both face 
the same epistemic gaps. That is, Mary's phenomenal concepts and Zombie Mary's quasiphenomenal concepts will be conceptually independent of their respective physical and functional concepts. In fact, I have shown earlier (section 3.2.1) that one can provide a reductive explanation of conceptual independence. Thus, if what we mean by the term epistemic situation is merely the epistemic or explanatory gaps that we face, and these gaps admit of a topic-neutral description, then both Mary and Zombie Mary will share the same epistemic situation: neither will be able to deduce a priori phenomenal (or quasi-phenomenal, in Zombie Mary's case) truths from PIT (see also Diaz-Leon 2010). In response, one might hold that epistemic gaps should be understood phenomenally. In that case, it is clear that the respective epistemic positions of Mary and Zombie Mary will differ. Nonetheless, this takes us back to the first horn of the dilemma that Chalmers' objection faces: namely, that $E$ should be given a phenomenal characterization. As I argued in the previous section, PCS has nothing to worry from such a characterization of E. ${ }^{10}$

${ }^{10}$ I was asked whether Chalmers can hold that zombies do not share our epistemic situation and at the same time maintain that our epistemic situation can be adequately described in topic-neutral terms. I do not see how. The only difference between zombies and us is the fact that zombies lack consciousness. But if that is the only difference, then our epistemic situation can differ from that of zombies only if an adequate characterization of our epistemic situation makes reference to phenomenal states. By assumption, however, our epistemic situation is given in topic-neutral terms. As a result, there cannot be any difference between our epistemic situation and that of zombies. As long as Chalmers admits that our epistemic situation can be characterized topic-neutrally, then I do not see how he can also maintain that zombies do not share our epistemic situation.

As a last resort, Chalmers might cite the content of certain utterances that zombies can make and argue that those utterances somehow demonstrate that zombies do not share our epistemic situation (See Chalmers 2010, p. 317ff.). For instance, he can argue that when a zombie utters, 'I am phenomenally conscious,' the zombie asserts something false. Yet, when we utter the same sentence we say something true. Therefore, we do not share zombies' epistemic situation. There are at least two problems with this argument. First, it is notoriously difficult to delineate the contents of zombies' utterances or beliefs. This difficulty is something to which Chalmers himself admits (ibid., p.317). In fact, it is precisely this difficulty that forces Chalmers to provide an additional argument in support of the claim that zombies do not share our epistemic situation. 'Therefore, if Chalmers' objection against PCS stands on contentious claims about the contents of zombie's beliefs, then his objection rests on rather shaky grounds. Furthermore, there are authors who argue against Chalmers' claim that zombies utter something false, when they utter 'I am phenomenally conscious' (see Balog 1999).

But there is a more serious issue here. What is at stake is whether the epistemic situation of zombies differs from our epistemic situation. In order for Chalmers' argument to show that, we must assume that the false sentence, 'I am phenomenally conscious,' uttered by a zombie, and the true sentence, 'I am phenomenally conscious,' uttered by us, are descriptions of both zombies' and our epistemic situations. It is only by demonstrating that there is a difference in the truth-values of those two utterances that Chalmers can 


\section{Conclusion}

In this paper, I have argued that Chalmers' objection against PCS is faced with a dilemma. Either our epistemic situation can be adequately captured by topic-neutral terms or not. The conclusion that I have reached is the following: Chalmers cannot both insist that $E$ should be given in topic-neutral terms and that our epistemic situation differs from that of zombies. It is either one or the other. If he gives up the latter claim, then the second horn of Chalmers' dilemma founders. If he gives up the former claim, then Chalmers' objection collapses to the claim that PCS is committed to the existence of an additional epistemic gap, either between $C$ and $E$ (if $C$ is characterized topic-neutrally) or PIT and $C$ (if $C$ is characterized phenomenally). But since PCS can provide justification for the claim that these additional gaps are compatible with physicalism, then PCS is safe. Unlike extant responses to Chalmers' objection, my response does not rest on a particular account of phenomenal concepts. Nor does it require a particular description of our epistemic situation: regardless of how our epistemic situation is described, Chalmers' objection does not undermine PCS.

maintain that we do not share the epistemic situation of zombies. For argument's sake, let us assume that Chalmers is right when he claims that the zombie utterance 'I am phenomenally conscious' is false. Now, if the false utterance (or belief) 'I am phenomenally conscious' is a description (be it a partial and incomplete one) of zombie's epistemic situation, then it cannot be a description of their epistemic situation that is given in topic-neutral terms. If it were, then we would have no reason to think that they have uttered something false. Hence, in order to conclude that a zombie utters something false when he or she utters 'I am phenomenally conscious' we must read that utterance in a phenomenal and not in a topic-neutral manner. But by doing so, we are assuming that our epistemic situation must be given in phenomenal terms: the zombie utterance 'I am phenomenally conscious' turns out to be false only if the term 'phenomenal consciousness' is not a topic-neutral term - i.e., it does not refer to a state that admits of a topic-neutral characterization.

Consequently, considerations of zombie utterances cannot show that their epistemic situation differs from ours, if we have already assumed that our epistemic situation can be adequately characterized in topic-neutral terms. In order to show that the two epistemic situations differ, we must assume a phenomenal description of our epistemic situation. But in this part of the essay, we have been assuming that our epistemic situation can be captured in topic-neutral terms. So Chalmers cannot use zombies' utterances to argue that the epistemic situation of zombies differs from our epistemic situation and that our epistemic situation can be adequately characterized in topic-neutral terms. (To be clear, I am not arguing that our epistemic situation can be adequately characterized in topic-neutral terms. My claim is a conditional one: if our epistemic situation can be captured in topic-neutral terms, then, pace Chalmers, zombies share our epistemic situation.) 


\section{References}

Balog, K. 1999. Conceivability, Possibility, and the Mind-body Problem. Philosophical Review 108: 497-528. . 2012. In Defense of the Phenomenal Concept Strategy. Philosophy and Phenomenological Research 82 (1): $1-23$.

Carruthers, Peter, Veillet, Bénédicte. 2007. The Phenomenal Concept Strategy, Journal of Consciousness Studies 14: 212-36.

Chalmers, D. 1996. The Conscious Mind: In Search of a Fundamental Theory. Oxford: Oxford University Press.

- 2006/2010. Phenomenal Concepts and the Explanatory Gap. In Chalmers, David, The Character of Consciousness. Oxford: Oxford University Press. (Originally, in Phenomenal Concepts and Phenomenal Knowledge: New Essays on Consciousness and Physicalism, edited by T. Alter and S. Walter. Oxford: Oxford University Press.)

Chalmers, D., and F. Jackson. 2001. Conceptual Analysis and Reductive Explanation. Philosophical Review 110:315-61.

Diaz-Leon, E. 2008. Defending the Phenomenal Concept Strategy. Australasian Journal of Philosophy 86 (4): 597-610.

. 2010. Can Phenomenal Concepts Explain the Explanatory Gap? Mind 119 (476): 933-51

Hill, C. 1997. Imaginability, Conceivability, Possibility and the Mind-Body Problem. Philosophical Studies 87:61-85.

Hill, C., and B. McLaughlin. 1999. There are Fewer Things in Reality Than are Dreamt of in Chalmers's Philosophy. Philosophy and Phenomenological Research

Levin, J. 2008. Taking Type-B Materialism Seriously. Mind \& Language 23 (4): 402-25.

Levine, J. 2006. Phenomenal Concepts and the Materialist Constraint. In Phenomenal Concepts and Phenomenal Knowledge: New Essays on Consciousness and Physicalism, edited by T. Alter and S. Walter. Oxford: Oxford University Press.

Loar, B. 1990/1997. Phenomenal States. In The Nature of Consciousness: Philosophical Debates, edited by N. Block, O. Flanagan and G. Güzeldere. Cambridge, MA: MIT Press.

- 2003. Qualia, Properties, Modality. In Philosophical Issues 13: Philosophy of Mind, edited by E. Sosa and E. Villanueva. Oxford: Blackwell.

- 2007. Thinking About Qualia. In Situating Semantics: Essays on the Philosophy of John Perry, edited by M. O'Rourke and C. Washington. Cambridge, MA: MIT Press.

Papineau, D. 2001. The Rise of Physicalism. In C. Gillet \& B. Loewer (Eds.), Physicalism and Its Discontents. Cambridge: Cambridge University Press.

2002. Thinking About Consciousness. Oxford: Oxford University Press.
. 2006. Phenomenal and Perceptual Concepts. In Phenomenal Concepts and Phenomenal Knowledge: New Essays on Consciousness and Physicalism, edited by T. Alter and S. Walter. Oxford: Oxford University Press.

Perry, J. 2001. Knowledge, Possibility, and Consciousness. Cambridge, MA: MIT Press.

Stoljar, D. 2005. Physicalism and Phenomenal Concepts. Mind \& Language 20 (5):469-95.

- 2010. Physicalism. New York: Routledge.

Sturgeon, S. 2000. Matters of Mind: Consciousness, Reason and Nature. New York: Routledge.

Tye, M. 1995. Ten Problems of Consciousness: A Representational Theory of the Phenomenal Mind. Cambridge, MA: MIT Press.

- 2000. Consciousness, Colour and Content. Cambridge, MA: MIT Press. 2009. Consciousness Revisited: Materialism Without Phenomenal Concepts. Cambridge, MA: MIT Press.

Yablo, S. 1992. Mental Causation. Philosophical Review 101: 245-280. 